\title{
A Reservoir Dynamic Model for Linear Optical Amplifiers
}

\author{
M. Salsi (1), A. Vannucci (1), A. Bononi (1), W. Mathlouthi (2), P. Lemieux (2) and L. A. Rusch (2)
}

1 : Dipartimento Di Ingenieria dell'Informazione, Università di Parma, I-43100, Italy. Email : salsi@tlc.unipr.it

2 : COPL, ECE Dept., Université Laval, Sainte-Foy, Québec G1K 7P4, Canada. Email : rusch@gel.ulaval.ca

\begin{abstract}
A novel state-variable dynamic model for semiconductor Linear Optical Amplifiers (LOA) is used to describe gain dynamics and stabilization properties of LOAs. Results from the model are verified against experiments.
\end{abstract}

\section{INTRODUCTION}

Semiconductor optical amplifiers (SOAs) are becoming key devices for many networking applications. A new SOA has recently been introduced [1] that achieves gain-stabilization through a series of internal vertical clamping laser cavities. Linear operation is thus guaranteed for a wide range of input powers, hence the name Linear Optical Amplifier (LOA) [1]. LOAs are ideal devices for burst-mode optical packet switching based metropolitan area networks [2]. Although LOA simulations have been performed by solving the detailed equations that govern the signal-amplifier interactions $[3,4]$, it would be highly desirable to have simple analytical and simulation tools able to quickly model LOAs in the design and analysis of an optical link. We present here, for the first time, an analytical simplified model for LOAs, and validate it by comparison with experiments. Such a Reservoir model stems from a similar state-variable approach that we recently adopted for SOAs [5].

\section{THE RESERVOIR MODEL FOR LOAS}

LOAs achieve gain stabilization thanks to a series of internal vertical lasing cavities that clamp the carrier density along the device. A numerical analysis of signals propagation in a LOA requires the joint solution of a set of differential equations [4] representing: signals propagation, the time adaptation of the vertical lasers, and the amplifier rate equation, describing the time evolution of the carrier density profile $N(z, t)$. In an SOA, the rate equation can be integrated along the active cavity length, by properly accounting for the amplifier material gain, scattering loss, and the generation of amplified spontaneous emission (ASE) noise, thus reducing the analysis to the solution of a single differential equation describing the time evolution of a state variable: the reservoir $r(t)$ [5]. Once $r(t)$ is known, the input-output relationship is readily determined for every input signal. Since a LOA is made of multiple cavities, the integration of the rate equation is done in each cavity separately, resulting in a multi-slice amplifier, each containing a vertical clamping laser. We model the amplifier geometry considering that the length $L$ of the active region is divided in $N_{C}$ independent cavities, each with a transverse area

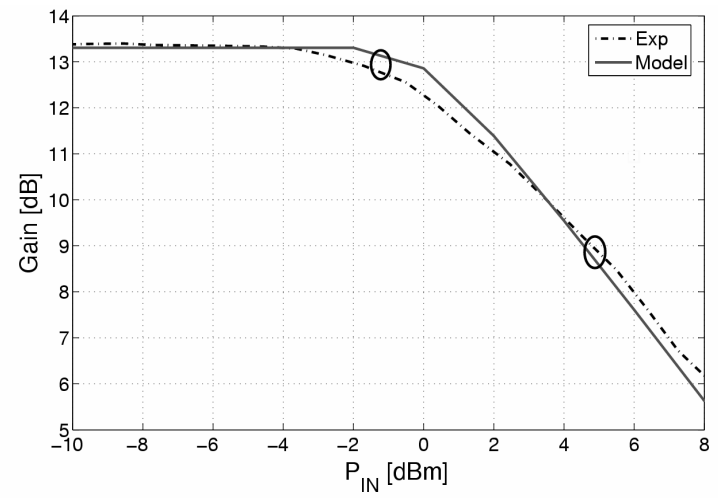

Fig. 1. LOA gain versus input power. Circles indicate the power levels used in Fig.3.

$A$ and length $l=L / N_{C}$. We define the reservoir of the $i^{\text {th }}$ cavity as:

$$
r^{i}(t)=A \int_{[l]} N(z, t) d z
$$

where $[l]$ means integration over the $i^{\text {th }}$ cavity and $\mathrm{z}$ is the signal propagation direction. For each cavity we consider the presence of signals $(k \in S)$ and ASE wavelengths $(k \in A)$, propagating in the forward direction only. The photon flux $Q_{L}^{i}$ of the $i^{\text {th }}$ clamping laser is independent of $\mathrm{z}$. For the $i^{\text {th }}$ cavity the reservoir dynamic equation is:

$$
\begin{aligned}
& \frac{d r^{i}}{d t}=\frac{I^{i}}{q}-\frac{r^{i}}{\tau^{i}}-\sum_{k \in S \cup A} Q_{k}^{I N, i}\left(G_{k}\left(r^{i}\right)-1\right)\left(1+\frac{\alpha l}{\ln \left(G_{k}\left(r^{i}\right)\right)}\right)(2) \\
& -4 \Delta v_{A S E} \sum_{j \in A} n_{s p, j}\left(r^{i}\right)\left(G_{j}\left(r^{i}\right)-1-\ln \left(G_{j}\left(r^{i}\right)\right)\right) . \\
& \left(1+\frac{\alpha l}{\ln \left(G_{j}\left(r^{i}\right)\right)}\right)-Q_{L}^{i} \ln \left(G_{L}\left(r^{i}\right)\right)
\end{aligned}
$$

where $q$ is the electron charge, $\tau^{i}$ is the fluorescence time, due to the carrier recombination rate, $I^{i}$ is the injected current, $\alpha$ is the scattering loss, $\Delta v_{A S E}$ is the ASE channel spacing, and $n_{s p, j}$ a length averaged spontaneous emission factor. The gain for the $k^{\text {th }}$ channel $G_{k}\left(r^{i}\right)=\exp \left\{B_{k} * r^{i}-A_{k}-\alpha l\right\}$ includes the scattering losses and is an exponential function of the reservoir through the pair of wavelength-dependent parameters $A_{k}$ and $B_{k}$, which are obtained by a linearization of the material gain $g_{m}(N)$ ([6] eq. 14). The same holds for the gain $G_{L}$ seen by the clamping laser. The photon flux $Q_{L}^{i}$, in each cavity, is found as [4]:

$$
\frac{d Q_{L}^{i}}{d t}=\frac{d}{v}\left(F^{i} r^{i}(t)-E^{i}-g_{t h r}^{i}\right) Q_{L}^{i}
$$




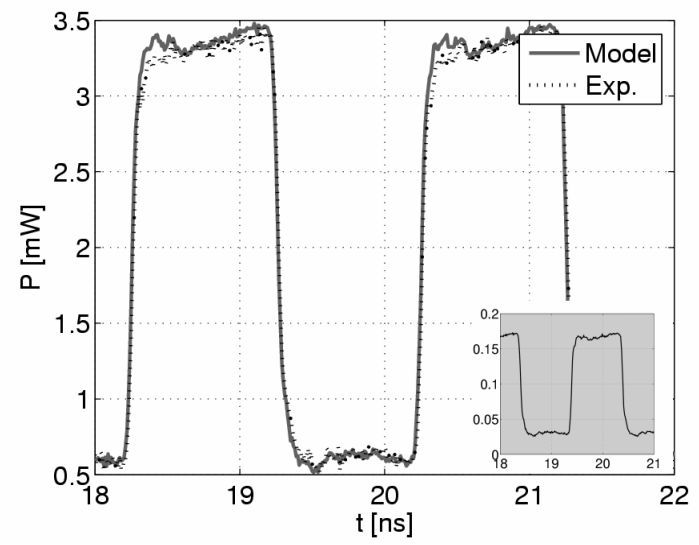

Fig. 2: LOA dynamic response; mean input power $-10 \mathrm{dBm}$ where $d$ is the length of the vertical cavity, $v$ is the group velocity, $F^{i}$ and $E^{i}$ are parameters similar to $A_{k}$ and $B_{k}$ but are referred to the vertical cavity that has an area and an overlap factor different from the ones seen by the signals. Equations (2) and (3) must be solved serially, for $i=1 \ldots N_{C}$, and finally the input-output gain for the $k$-th channel is the product of the gains provided by each LOA cavity.

\section{EXPERIMENTAL RESULTS}

We now compare the simulation results of our model with static and dynamic measurements that were performed on a Finisar LOA. First, we measured the gain of the LOA versus input power using an input $\mathrm{CW}$ signal at $1550 \mathrm{~nm}$. Measurements are shown in Fig.1 along with simulations from the Reservoir model, and a good fit is observed. Fig.2 shows the dynamic response of the LOA to a square-wave input, visible in the inset. The input mean power is $-10 \mathrm{dBm}$, ensuring that the device is in a state where all the vertical lasers are ON. In this situation our model, fed with the measured input signal, well fits experimental results. The transmitter operates at $1 \mathrm{~Gb} / \mathrm{s}$, and bandwidth limitations are due to the transmitter circuitry, as visible in the input signal inset.

Fig. 3 shows the LOA behaviour in saturation. The amplifier is fed with a mean input power of $3 \mathrm{dBm}$ and the extinction ratio is kept at $7 \mathrm{~dB}$ so that the input power levels are those marked in Fig.1. This way, we can test the gain clamping model when switching between near-linear and saturated modes of operation. When the same input signal is used for the simulations, the Reservoir model well captures the transient behaviour of the amplifier. The large power overshoots are due to the clamping lasers turned momentarily off by the very large step transition in the input. The onset of gainclamping is achieved after a small fraction of the bit duration, eventually reaching steady power on the mark level. A markzero transition in our setup makes the LOA operate in the near-linear region in Fig.1, where the clamping lasers tend to oscillate in a metastable state. This produces ripples on the zeros which are averaged out by the receiver. An unfiltered simulation is added in Fig. 3 to show such remarkable feature,

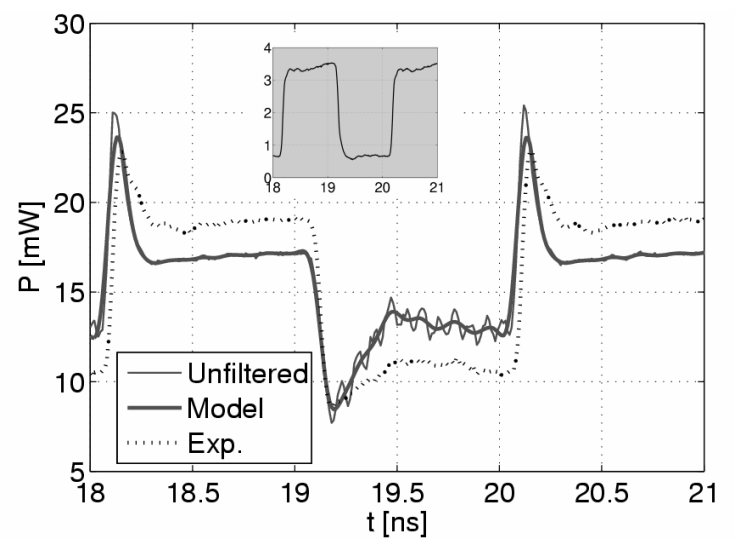

Fig. 3: LOA dynamic response; mean input power $+3 \mathrm{dBm}$. appearing only in this most critical situation. The residual offset of power levels in Fig.3 is quantified by the mismatch of static gain curves in Fig.1, which still require finer fitting.

\section{CONCLUSION}

A novel Reservoir LOA dynamic model was introduced. For the first time, simulation results from a LOA model are checked against experimental measurements performed on a commercial device, finding very good agreement. The purpose of the proposed model is twofold. First, it reduces the equations describing the LOA to a minimal complexity, using a single state variable for each independent amplifier cavity; hence, it is suitable for performing fast simulations in optical network design. Second, the model gives analytical insight and a monitoring of the LOA transversal clamping lasers, otherwise not accessible from the device. In addition, thanks to the reservoir approach, there is no need for solving joint differential equations for signals and carriers, hence the low computation times are little affected by the number of channels. The model is thus a good tool in WDM large optical networks simulations.

\section{ACKNOWLEDGEMENTS}

Support through the OSATE project funded by the Italian Ministry for Research is acknowledged.

\section{REFERENCES}

[1] D. A. Francis, et al., "A single-chip linear optical amplifier," $O F C$ '01, PD13, 2001.

[2] I. M. White, et al., "Demonstration and system analysis of the HORNET architecture," IEEE J. Lightwave Technology, vol. 21, pp. 2489-2498, 2003

[3] J. Oksanen, et al., "On crosstalk and noise in an optical amplifier with gain clamping by vertical laser field," IEEE J. Lightwave Technology, vol. 46, pp. 1914-1919, 2003.

[4] C.-Y. Jin, et al., "Numerical and theoretical analysis of the crosstalk in linear optical amplifiers," IEEE J. Quantum Electronics, vol. 41, pp. 636-641, 2005.

[5] W. Mathlouthi et al., "Fast and Efficient Dynamic WDM Semiconductor Optical Amplifier Model," IEEE J. Lightwave Technology, submitted.

[6] M. J. Connelly, "Wideband Semiconductor Optical Amplifier SteadyState Numerical Model," IEEE J. Quantum Electronics, vol. 37, pp. 439-447, 2001. 